\title{
CRESCIMENTO DE MUDAS DE GARAPA EM RESPOSTA À CALAGEM E AO FÓSFORO'
}

\author{
Keli Cristina de Oliveira Gomes ${ }^{2}$, Haroldo Nogueira de Paiva ${ }^{3}$, Júlio César Lima Neves ${ }^{4}$, Nairam Félix de \\ Barros $^{4}$, Sérgio Ricardo Silva ${ }^{4}$
}

\begin{abstract}
RESUMO - Este trabalho teve por objetivo avaliar o desempenho inicial da garapa (Apuleia leiocarpa (Vogel) Macbride) em condições diferenciadas de disponibilidade de fósforo $(\mathrm{P})$ no solo e de saturação por bases (V). Os tratamentos foram arranjados em esquema fatorial combinando seis doses de $\mathrm{P}(0,100,200,300,400$ e $500 \mathrm{mg}$ dm-3) e cinco níveis de calagem empregando o critério da saturação por bases, para cálculo (original $=24,40$, 50, 60 e 70\%). O delineamento foi em blocos ao acaso com cinco repetições. Aos 120 dias após a semeadura, mediram-se a altura e diâmetro do coleto de todas as plantas, as quais foram colhidas, separadas em raiz e parte aérea e secas, para obtenção de massa seca constante. Os resultados permitiram a conclusão de que o nível de calagem recomendado para a formação de mudas de garapa é aquele que proporciona a elevação da saturação por bases a $60 \%$, sendo, nessas condições, a dose recomendada de $\mathrm{P}$ de $54 \mathrm{mg} \mathrm{dm}^{-3}$. O nível crítico de P no solo foi influenciado pelo valor da saturação por bases, variando de $6(\mathrm{~V}=60 \%)$ a $33 \mathrm{mg} \mathrm{dm}^{-3}(\mathrm{~V}=40 \%)$. O nível crítico de $\mathrm{P}$ na matéria seca da parte aérea também foi influenciado pela saturação por bases, variando de $0,11(\mathrm{~V}=60 \%)$ a $0,18 \mathrm{dag} \mathrm{kg}^{-1}(\mathrm{~V}=50 \%)$.
\end{abstract}

Palavras-chave: Apuleia leiocarpa, garapa, saturação por bases e fósforo.

\section{GROWING OF GARAPA SEEDLING IN RESPONSE TO LIME AND PHOSPHORUS}

\begin{abstract}
This study aimed to evaluate the initial performance of Apuleia leiocarpa (Vogel) Macbride, in variable conditions of phosphorus $(P)$ availability and base saturation $(V)$ in the soil. The treatments were arranged in a factorial design of $6 \mathrm{P}$ levels $(0 ; 100 ; 200 ; 300 ; 400$ and $500 \mathrm{mg} \mathrm{dm}-3)$ and 5 base saturation levels (original $=24 ; 40 ; 50 ; 60$ and 70\%), in complete randomized blocks with five replicates. Within one hundred twenty days after seed sowing, plant heights and basal stalk diameters were measured; plants were harvested, separated into roots and shoots, and dried to constant mass. The results showed that the maximum recommended lime rate for A. leiocarpa is $60 \%$ of base saturation. Under these conditions the recommended $P$ dose is 54 $m g \mathrm{dm}^{-3}$. The $P$ critical level in the soil was influenced by the base saturation, ranging from $6(V=60 \%)$ to $33 \mathrm{mg} \mathrm{dm}^{-3}(V=40 \%)$. The P critical level in the shoot dry matter was also influenced by base saturation, varying from $0.11(\mathrm{~V}=60 \%)$ to $0.18 \mathrm{dag} \mathrm{kg}^{-1}(\mathrm{~V}=50 \%)$.
\end{abstract}

Keywords:Apuleia leiocarpa, "garapa", base saturation level and phosphorus.

${ }^{1}$ Recebido em 17.03.2006 e aceito para publicação em 19.05.2008.

${ }^{2}$ Mestre em Ciência Florestal pela UFV. E-mail : <keli_cog@yahoo.com.br>.

${ }^{3}$ Departamento de Engenharia Florestal da Universidade Federal de Viçosa (UFV) - Viçosa-MG. E-mail : <hnpaiva@ufv.br>.

${ }^{4}$ Departamento de Solos da UFV. E-mail : <julio_n2003@yahoo.com.br>. 


\section{INTRODUÇÃO}

A Mata Atlântica, declarada "patrimônio nacional" pela Constituição Federal de 1988, é o bioma mais debatido e conhecido do Brasil. Possui grande estrutura legal de proteção, nos níveis federal, estadual e municipal. Já ocupou cerca de um milhão de $\mathrm{km}^{2}$, e hoje está reduzida a $10 \%$ da área original (BRANDÃO, 1997). A Zona da Mata de Minas Gerais, embora esteja incluída no bioma Mata Atlântica, apresenta cobertura florestal formada por pastagens e campos de cultivos com fragmentos florestais nativos esparsamente distribuídos (MEIRA NETO e SILVA, 1995).

A maioria das espécies florestais que ocorrem na Mata Atlântica apresenta de média a alta demanda nutricional. Assim, a prática de adubação tem sido fundamental não só para a produção de mudas de boa qualidade silvicultural, mas também para que as plantações florestais alcancem níveis adequados de crescimento no campo (GONÇALVES et al., 2000). Segundo esses mesmos autores, devido à grande diversidade de espécies e, conseqüentemente, de exigências nutricionais, as recomendações de adubação no campo têm sido feitas para assegurar o suprimento de nutrientes para as espécies mais exigentes, de forma que as demais também tenham suas demandas nutricionais atendidas. Portanto, pesquisas que visem avaliar o comportamento de espécies florestais que possam apresentar bom desempenho na região e que possuam características interessantes a diversas finalidades de uso são de grande valia. Nesse sentido, trabalhos como o de Meira Neto (1997), que realizou a caracterização florística, estrutural e ambiental de estratos arbóreos e herbáceo-arbustivo de uma Floresta Estacional Semidecidual em Viçosa, podem embasar, em parte, estudos dessa natureza.

Apuleia leiocarpa (garapa) é uma espécie arbórea da família Fabaceae - Caesalpinoideae, com 25 a 35 $\mathrm{m}$ de altura e 60 a $90 \mathrm{~cm}$ de diâmetro. Apresenta crescimento lento a moderado, ocorrendo desde o Estado do Pará até o Rio Grande do Sul, em floresta pluvial (LORENZI, 1992). A espécie possui comportamento de pioneira indiferente à secundária tardia e regeneração abundante nas florestas secundárias e povoa, com facilidade, as capoeiras e roças abandonadas. É considerada padrão de terrenos secos e profundos, sempre encontrada nos lugares altos. É recomendada para reposição de mata ciliar em locais sem inundação (CARVALHO, 1994). Ainda, foi listada como mais importante em pelo menos três levantamentos fitossociológicos em matas mesófilas em Minas Gerais (MEIRA NETO et al., 1997).
Este trabalho foi realizado com o objetivo de avaliar a influência de níveis de calagem e doses de fósforo no crescimento da garapa.

\section{MATERIAL E MÉTODOS}

Esta pesquisa foi desenvolvida no Viveiro de Pesquisa do Departamento de Engenharia Florestal, pertencente à Universidade Federal de Viçosa, em Viçosa, MG.

Os tratamentos foram representados por um fatorial combinando seis doses de $\mathrm{P}(0 ; 100 ; 200 ; 300 ; 400$; e 500

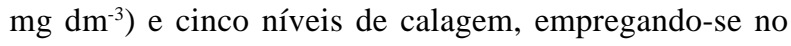
cálculo desta o método da saturação por bases (original $=24,40,50,60$ e 70\%). Os tratamentos foram dispostos em delineamento em blocos ao acaso, com cinco repetições. A parcela experimental foi constituída por um vaso de polietileno rígido, contendo $2 \mathrm{dm}^{3}$ de solo. A espécie utilizada foi garapa (Apuleia leiocarpa (Vogel) Macbride).

Como substrato, utilizou-se amostra coletada na camada de 0-20 cm de profundidade de um Latossolo Vermelho Distrófico, textura argilosa, com as seguintes características granulométricas, em g/kg: argila $=450$; silte $=90 ;$ areia grossa $=210$ e areia fina $=250$; e químicas: $\mathrm{pH}=5,1 ; \mathrm{P}\left(\mathrm{mg} \mathrm{dm}^{-3}\right)=4,8 ; \mathrm{K}\left(\mathrm{mg} \mathrm{dm}^{-3}\right)=68 ; \mathrm{Al} 3+$ $\left(\mathrm{cmol}_{\mathrm{c}} \mathrm{dm}^{-3}\right)=0,1 ; \mathrm{Ca}^{2+}\left(\mathrm{cmol}_{\mathrm{c}} \mathrm{dm}^{-3}\right)=0,9 ; \mathrm{Mg}^{2+}\left(\mathrm{cmol}_{\mathrm{c}}\right.$ $\left.\mathrm{dm}^{-3}\right)=0,4 ; \mathrm{H}+\mathrm{Al}\left(\mathrm{cmol}_{\mathrm{c}} \mathrm{dm}^{-3}\right)=4,6 ; \mathrm{SB}\left(\mathrm{cmol}_{\mathrm{c}} \mathrm{dm}^{-3}\right)$ $=1,47 ; \mathrm{CTC}(\mathrm{t})\left(\mathrm{cmol} \mathrm{dm}_{\mathrm{c}} \mathrm{dm}^{-3}\right)=1,57 ; \mathrm{CTC}(\mathrm{T})\left(\mathrm{cmol}_{\mathrm{c}} \mathrm{dm}^{-3}\right)$ $=6,07 ; \mathrm{V}(\%)=24 ; \mathrm{e} \mathrm{MO}(\mathrm{dag} / \mathrm{kg})=2,46$. A seguir, o solo recebeu as doses de corretivos, sendo empregada uma mistura de $\mathrm{CaCO}_{3}$ e $\mathrm{MgCO}_{3}$, na relação estequiométrica de 4:1. Depois da aplicação dos corretivos, seguiu-se um período de incubação de 30 dias, mantendo o teor de água próximo à capacidade de campo, inclusive no tratamento que não recebeu calcário.

$\mathrm{Na}$ aplicação das doses de $\mathrm{P}$, dois dias antes da semeadura fez-se uma adubação de manutenção, aplicada via solução, constituída de $100 \mathrm{mg} \mathrm{dm}^{-3} \mathrm{de} \mathrm{N}, 100 \mathrm{mg}$ $\mathrm{dm}^{-3}$ de $\mathrm{K}$ e $40 \mathrm{mg} \mathrm{dm}^{-3}$ de $\mathrm{S}$, empregando como fontes desses nutrientes os sais $\mathrm{NH}_{4} \mathrm{NO}_{3}, \mathrm{KCl} \mathrm{e} \mathrm{K}_{2} \mathrm{SO}_{4}$, conforme sugerido por Passos (1994). Ainda, foi feita a aplicação de uma solução de micronutrientes, nas seguintes doses: $\mathrm{B}=0,81 \mathrm{mg} \mathrm{dm}^{-3}\left(\mathrm{H}_{3} \mathrm{BO}_{3}\right), \mathrm{Mn}=3,66 \mathrm{mg} \mathrm{dm}^{-3}\left(\mathrm{MnCl}_{2} \cdot \mathrm{H}_{2} \mathrm{O}\right)$, $\mathrm{Zn}=4,00 \mathrm{mg} \mathrm{dm}^{-3}\left(\mathrm{ZnSO}_{4} \cdot 7 \mathrm{H}_{2} \mathrm{O}\right), \mathrm{Cu}=1,33 \mathrm{mg} \mathrm{dm}^{-3}$ $\left(\mathrm{CuSO}_{4} \cdot 5 \mathrm{H}_{2} \mathrm{O}\right)$ e $\mathrm{Mo}=0,15 \mathrm{mg} \mathrm{dm}^{-3}\left(\left(\mathrm{NH}_{4}\right)_{6} \mathrm{Mo}_{7} \mathrm{O}_{24} \cdot 4 \mathrm{H}_{2} \mathrm{O}\right)$, de acordo com Alvarez V. (1974).

Foram realizadas, ainda, mais três adubações nitrogenadas, aos 35, 70 e 105 dias após a semeadura, 
na dose de $20 \mathrm{mg} \mathrm{dm}^{-3}$ de $\mathrm{N}$, por aplicação, utilizandose como fontes do nutriente, nas duas primeiras épocas, o $\mathrm{NH}_{4} \mathrm{NO}_{3} \mathrm{e}$, na última, o $\mathrm{KNO}_{3}$. Nessa última aplicação, juntamente com o $\mathrm{N}$ foram adicionados $55,8 \mathrm{mg} \mathrm{dm}^{-3}$ de K, conforme sugerido por Garcia (1986). Durante o período experimental, a umidade do solo foi mantida próxima a $60 \%$ da capacidade máxima de retenção de água, utilizando-se água deionizada.

Aos 120 dias após a semeadura, foram medidos a altura e o diâmetro do coleto de todas as plantas, encerrando-se o experimento. As plantas foram separadas em raiz e parte aérea, lavadas com água destilada e secadas em estufa com circulação forçada de ar, a uma temperatura próxima de $70^{\circ} \mathrm{C}$ até atingir massa constante. O material vegetal seco e moído foi mineralizado, pela mistura nítrico-perclórica, e nos extratos procedeuse à determinação de $\mathrm{P}$. O P foi dosado por colorimetria pelo método da Vitamina C (BRAGA; DEFELIPO, 1974) em espectrofotômetro a $725 \mathrm{~nm}$.

Ao final do experimento, o solo contido nos vasos foi seco ao ar e peneirado, sendo, então, submetido à análise de P disponível, pelo Mehlich -1 (EMBRAPA, 1997).

Os dados foram submetidos à análise de variância, usando-se o programa SAEG (EUCLYDES, 1983). O coeficiente de correlação de Pearson foi calculado para avaliar a correlação entre a matéria seca da parte aérea e as variáveis altura, diâmetro do coleto e matéria seca de raiz. Foram ajustadas equações de regressão, para os valores de altura, diâmetro do coleto, peso de matéria seca, teor de $\mathrm{P}$ na parte aérea das plantas e $\mathrm{P}$ disponível no solo em função das doses de $\mathrm{P}$ aplicadas, dentro de cada nível de calagem. As doses recomendáveis de $\mathrm{P}$ foram aquelas responsáveis pela obtenção de $90 \%$ da produção máxima estimada de matéria seca da parte aérea. Substituindo essas doses nas equações obtidas para o $\mathrm{P}$ recuperado, pelo Mehlich-1, em função do $\mathrm{P}$ adicionado, foram obtidos os níveis críticos de $\mathrm{P}$ no solo. Já na parte aérea os níveis críticos de $\mathrm{P}$ foram resultantes da substituição das doses recomendáveis nas equações, relacionando os teores de $\mathrm{P}$ nessa parte das plantas em função das doses adicionadas.

\section{RESULTADOS E DISCUSSÃO}

Houve pequena resposta das mudas de garapa à correção do solo, em razão, provavelmente, de os teores originais de $\mathrm{Ca}\left(0,9 \mathrm{cmol}_{\mathrm{c}} \mathrm{dm}^{-3}\right)$ e de $\mathrm{Mg}\left(0,4 \mathrm{cmol}_{\mathrm{c}}\right.$ $\mathrm{dm}^{-3}$ ) no solo terem sido suficientes para suprir as necessidades das plantas nessa fase. Deve-se ressaltar que os menores valores de produção de matéria seca, altura e diâmetro de plantas foram obtidos no maior nível de saturação por bases $(\mathrm{V}=70 \%)$. Tal fato pode ser atribuído ao provável aumento excessivo do $\mathrm{pH}$ do solo nesse tratamento, acarretando, assim, a precipitação e, ou, menor disponibilidade para as plantas de micronutrientes como Zn e Mn, e comprometendo o estado nutricional e, conseqüentemente, o crescimento das plantas. Segundo Marschner(1995), com o aumento do pH do solo decresce a disponibilidade de zinco e manganês mais do que a de quaisquer outros nutrientes minerais, sendo alto o risco de deficiência depois da calagem.

Houve aumento da altura $(\mathrm{H})$, do diâmetro do coleto (D), da produção de matéria seca da parte aérea (MSPA) e de raízes (MSR) em resposta à adubação fosfatada nos tratamentos com saturação por bases menores que $70 \%$. Para esse nível ( $\mathrm{V}=70 \%)$ somente houve diferença significativa, em relação às doses de $\mathrm{P}$ aplicadas, para $\mathrm{D}$, ocorrendo aumento linear em resposta às doses de P (Figura 1). Observa-se que maiores doses de P são especialmente importantes para níveis intermediários de saturação por bases ( $\mathrm{V}=40$ e $50 \%$ ), quando o objetivo é promover incremento máximo das características avaliadas.

Constatou-se que a MSPA apresentou correlação de Pearson significativa $(\rho<0,01)$ e positiva com a $\mathrm{H}(\mathrm{r}=0,94), \mathrm{D}(\mathrm{r}=0,88)$ e MSR $(\mathrm{r}=0,94)$, o que explica a alta similaridade entre as equações de regressão ajustadas para essas características avaliadas (Figura 1).

A relação raiz/parte aérea (R/PA), em todos os níveis de saturação por bases, exceto no nível de 70\%, apresentou resposta negativa à aplicação de $\mathrm{P}$ (Figura 1 ). O maior valor estimado $(0,39)$ foi atingido no nível de $50 \%$ de saturação por bases. O decréscimo da relação R/PA, com o aumento da disponibilidade de P, também foi observado em diferentes espécies de eucalipto (SILVA, 2000; FURTINI NETO, 1994). De maneira similar, para copaíba, a partição da matéria seca entre a parte aérea e o sistema radicular, sob a omissão de $\mathrm{P}$ e de $\mathrm{N}$, resultou em maior mobilização de reservas para as raízes (DUBOC et al., 1996). Em solos com baixa disponibilidade de $\mathrm{NeP}$, o crescimento radicular é favorecido (MARSCHNER et al., 1996), como estratégia para absorver o máximo de nutrientes (CLARKSON, 1985). No entanto, segundo Araújo (2000), o maior destino de carbono às raízes sob baixo $\mathrm{P}$, tanto para a produção de biomassa quanto para a respiração de manutenção, pode constituir fator limitante ao crescimento vegetal como um todo, como foi observado neste trabalho. 

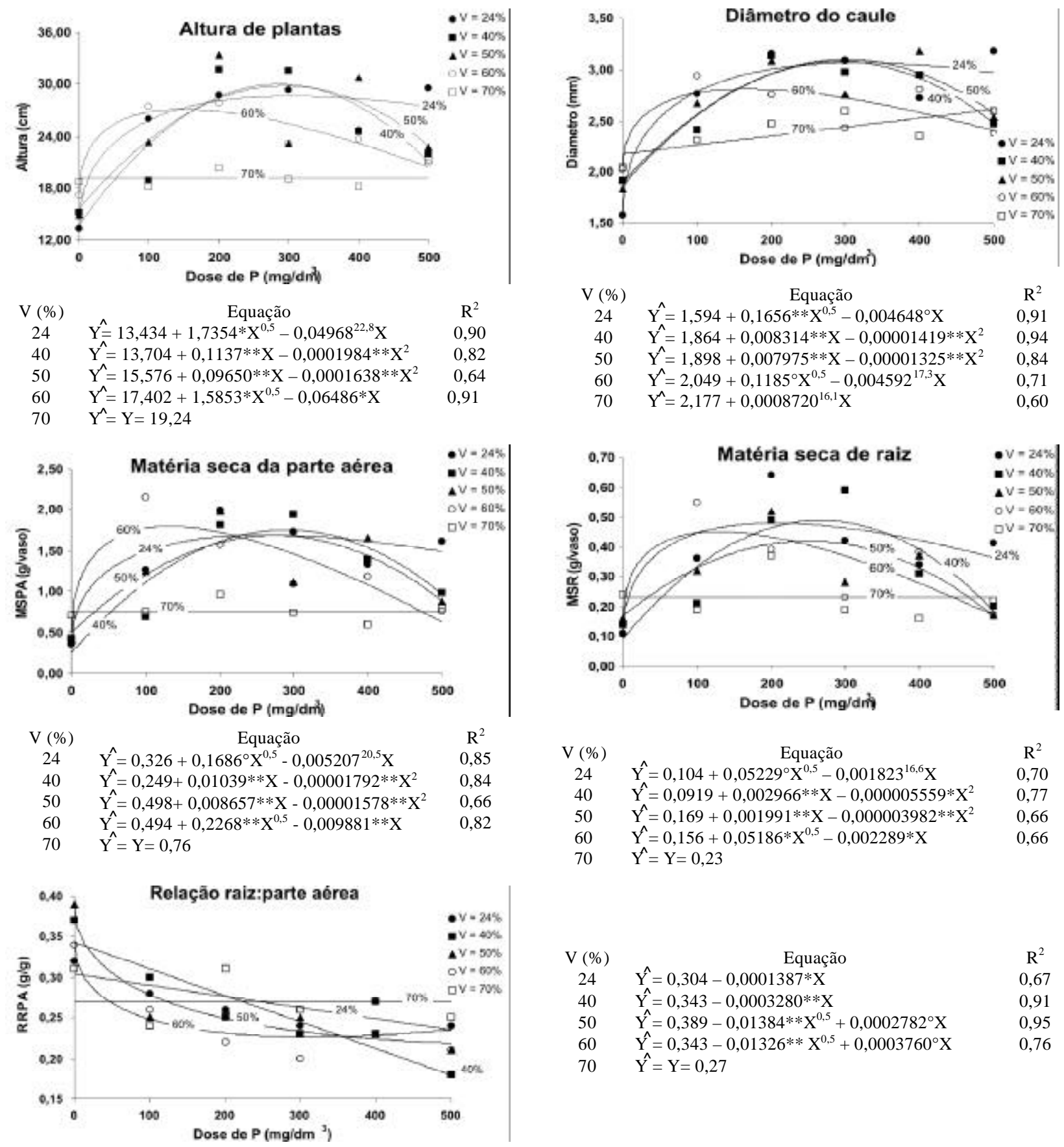
$\mathrm{V}(\%) \quad$ Equação $\quad \mathrm{R}^{2}$
$24 \hat{Y}=0,304-0,0001387 * X \quad 0,67$
$40 \hat{Y}=0,343-0,0003280 * * X \quad 0,91$
$50 \hat{Y}=0,389-0,01384 * * X^{0,5}+0,0002782^{\circ} \mathrm{X} \quad 0,95$
$60 \hat{\mathrm{Y}}=0,343-0,01326^{* *} \mathrm{X}^{0,5}+0,0003760^{\circ} \mathrm{X} \quad 0,76$
$70 \hat{\mathrm{Y}}=\mathrm{Y}=0,27$

Coeficiente significativo a $1 \%(* *), 5 \%(*)$ e $10 \%\left({ }^{\circ}\right)$ de probabilidade.

Figura 1 - Equações de regressão para altura, diâmetro do coleto, matéria seca da parte aérea e de raiz e relação raiz/parte aérea como variáveis dependentes das doses de $\mathrm{P}$ nos diferentes níveis de saturação por bases, para produção de mudas de garapa (Apuleia leiocarpa).

Figure 1 -Regression equations for height, stem diameter, dry matter productions of the aerial part and $P$ concentrations is shoot dry matter as dependent variables of $P$ doses in different base for garapa (Apuleia leiocarpa) seedling production.

R. Árvore, Viçosa-MG, v.32, n.3, p.387-394, 2008 
Verificou-se que o rendimento de MSPA, para atingir $90 \%$ da máxima produção, nos tratamentos com $\mathrm{V}=$ $60 \%\left(1,63 \mathrm{~g} \mathrm{vaso}^{-1}\right)$ foi $3,16 \%$ superior em relação a $\mathrm{V}=40 \%\left(1,58 \mathrm{~g} \mathrm{vaso}^{-1}\right)$ e $7,24 \%$ superior quando $\mathrm{V}=24$ e $50 \%\left(1,52 \mathrm{~g} \mathrm{vaso}^{-1}\right)$. Portanto, a despeito da pequena diferença entre esses valores, a calagem recomendada para garapa, nessas condições, é aquela que proporcione a elevação da saturação por bases a $60 \%$. Cruz et al. (2004), trabalhando com mudas de ipê-roxo (Tabebuia impetiginosa), em Latossolo Vermelho-Amarelo Distrófico, recomendaram elevar a saturação por bases para $50 \%$, visando produzir mudas de melhor qualidade; resultado semelhante encontrado para mudas de angico-vermelho (Anadenanthera macrocarpa) por Bernardino et al. (2005), ao passo que Bernardino et al. (2007) recomendaram elevar a saturação por bases para $60 \%$ para a produção de mudas de jacarandá-da-bahia (Dalbergia nigra), utilizando como substrato amostras de Argissolo. Em mudas de Eucalyptus grandis X E. urophylla, Silva et al. (2007) constataram que a elevação da saturação por bases para 50\% proporcionou o melhor crescimento destas, em termos de produção de matéria seca de parte aérea, à época da aplicação de $150 \mathrm{mg} \mathrm{P} \mathrm{dm}^{-3}$.

Houve interação significativa para saturação por bases (V) versus doses de $\mathrm{P}$, portanto, para cada nível de $\mathrm{V}$ estudado, houve uma dose de $\mathrm{P}$ que proporcionou a melhor produção de biomassa. A dose de $\mathrm{P}$ que proporcionou $90 \%$ de produção máxima, com base na produção de matéria seca da parte aérea, variou entre $54 \mathrm{mg} \mathrm{dm}^{-3}$ no nível de saturação por bases de $60 \%$ e $191 \mathrm{mg} \mathrm{dm}^{-3}$ no nível de 40\% (Quadro 1). Portanto, a recomendação de adubação fosfatada para a formação de mudas de garapa é de $54 \mathrm{mg} \mathrm{dm}^{-3}$ de $\mathrm{P}$, juntamente com a aplicação de calcário que eleve a saturação por bases a $60 \%$, uma vez que nesse nível de calagem foi obtida a maior produção de MSPA. Reis et al. (1997), usando substrato física e quimicamente semelhante ao deste trabalho, recomendaram a dose de $P$ equivalente a $250 \mathrm{mg} \mathrm{dm}^{-3}$ para produção de mudas de jacarandáda-bahia, enquanto Dias et al. (1991) recomendaram $276 \mathrm{mg} \mathrm{dm}^{-3}$ de $\mathrm{P}$ para a produção de mudas de taxibranco. Ceconi et al. (2006) verificaram que a dose de $360 \mathrm{mg} \mathrm{dm}^{-3}$ resultou no maior crescimento de mudas de açoita-cavalo, enquanto Ceconi et al. (2007) concluíram que o melhor crescimento das mudas de Ilex paraguariensis ocorreu com as doses de 360 a 450 $\mathrm{mg} \mathrm{dm}^{-3}$. Fernandes et al. (2007) constataram aumento linear da produção de matéria seca das mudas de freijó com as doses de $\mathrm{P}$, que variaram de 0 a 450 $\mathrm{mg} \mathrm{dm}{ }^{-3}$. A variação da dose de $\mathrm{P}$ recomendada para as diferentes espécies indica que a garapa é uma planta de ambiente de baixa fertilidade, quando comparada, por exemplo, com as espécies citadas anteriormente.

Quadro 1 - Doses de P recomendadas e níveis críticos no solo e na parte aérea das plantas de garapa, nos diferentes níveis de saturação por bases

Table 1 -Recommended P doses and critical levels in the soil and aerial part of garapa plants, at the different base saturation levels

\begin{tabular}{cccc}
\hline $\begin{array}{c}\text { Saturação por } \\
\text { bases }\end{array}$ & $\begin{array}{c}\text { Dose de P } \\
\text { recomendada }\end{array}$ & Nível crítico de P \\
\hline$(\%)$ & $\left(\mathrm{mg} \mathrm{dm}^{-3}\right)$ & $\begin{array}{c}\text { No solo } \\
\left(\mathrm{mg} \mathrm{dm}^{-3}\right)\end{array}$ & $\begin{array}{c}\text { Na parte aérea } \\
\left(\text { dag kg }^{-1}\right)\end{array}$ \\
\hline 24 & 109 & 16,83 & 0,13 \\
40 & 191 & 32,50 & 0,15 \\
50 & 172 & 30,00 & 0,18 \\
60 & 54 & 6,08 & 0,11 \\
\hline Média & 131,5 & 21,35 & 0,14 \\
\hline
\end{tabular}

Houve aumento linear do $\mathrm{P}$ disponível (P-disp.) no solo e aumento exponencial do teor de $\mathrm{P}$ na matéria seca da parte aérea (P-mspa) em resposta à adubação fosfatada (Figura 2). Segundo Costa (1998), quando se eleva a dose de P aplicada ao solo, ocorre aumento na difusão desse nutriente, devido à saturação progressiva da superfície de adsorção, o que resulta no aumento da concentração de $\mathrm{P}$ na solução do solo e em consequiente aumento na absorção do nutriente.

As equações ajustadas para P-disp. e P-mspa permitiram a obtenção de níveis críticos do nutriente no solo e na matéria seca da parte aérea (Quadro 1). O nível crítico de $\mathrm{P}$ no solo foi relativamente elevado na a maioria dos tratamentos, variando de $6(\mathrm{~V}=60 \%)$ a $33 \mathrm{mg} \mathrm{dm}^{-3}(\mathrm{~V}=40 \%)$. Elevados valores críticos de P no solo, na fase de formação de mudas, têm sido determinados para diferentes espécies florestais, como eucalipto (60 $\mathrm{mg} \mathrm{dm}^{-3}$ ) (BARROS et al., 1982), algaroba (37,9 $\left.\mathrm{mg} \mathrm{dm}^{-3}\right)$ (PASSOS, 1994), Eucalyptus grandis X E. urophylla $\left(27,4 \mathrm{mg} \mathrm{dm}^{3}\right)$ (SILVAet al., 2007) e outras leguminosas, como Sclerobium paniculatum $(26,1 \mathrm{mg}$ $\left.\mathrm{dm}^{-3}\right)$, Acacia holocericea $\left(18,6 \mathrm{mg} \mathrm{dm}^{-3}\right)$ e Mimosa tenuiflora (49,5 $\mathrm{mg} \mathrm{dm}^{-3}$ ), respectivamente, em estudos conduzidos por Dias et al.(1991), Fernández et al.(1996) e Balieiro et al. (2001). 

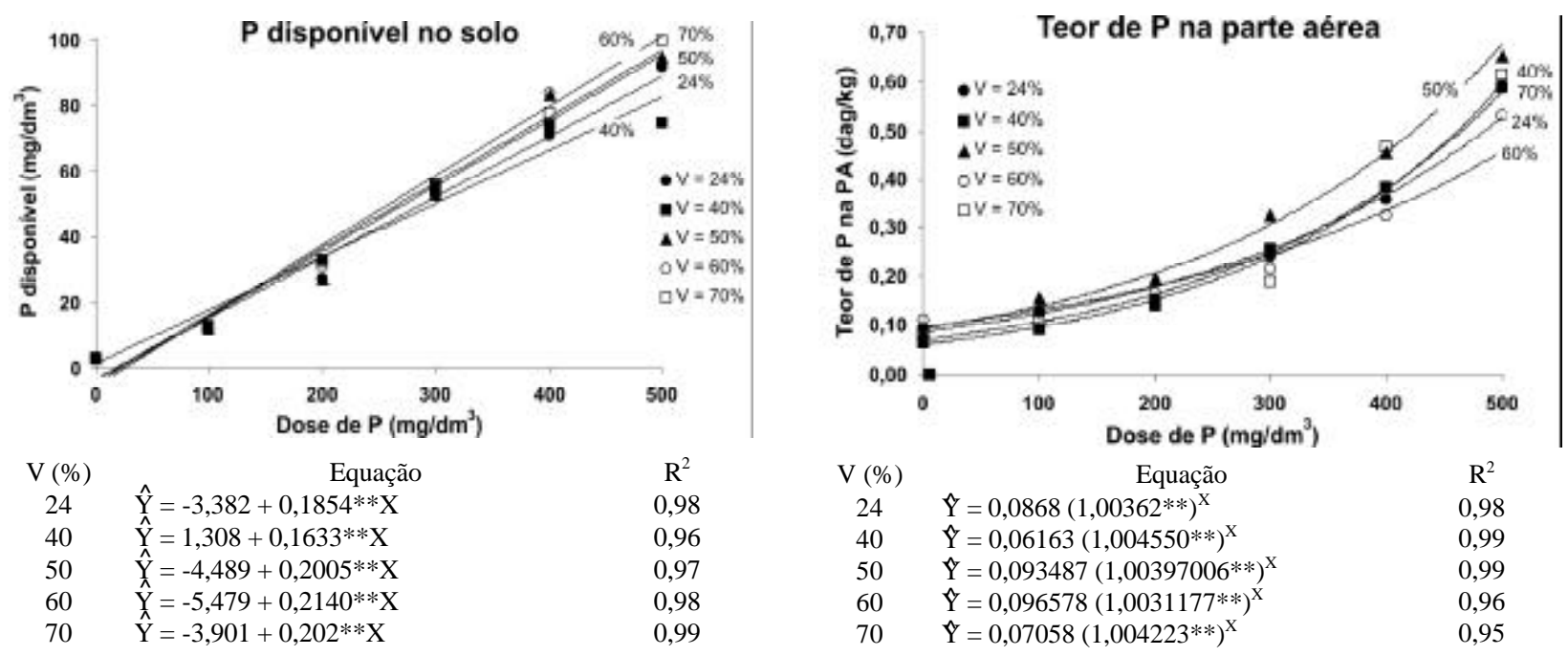

** Coeficiente significativo a $1 \%$ de probabilidade.

Figura 2 - Equações de regressão ajustadas de teor de P disponível no solo pelo extrator Mehlich-1 (P-disp.) e teor de P na matéria seca da parte aérea (P-mspa), em função das doses de P aplicadas ao substrato.

Figure 2 - Regression equations adjusted to dry matter productions of the aerial part (Mspa), concentrations of soil-available $P$ in the Mehlich-1 extract ( $P$-disp) and P concentrations in shoot dry matter $(P-m s)$, as a function of $P$ doses applied to the substratum.

Nos diferentes níveis de saturação por bases, o nível crítico de P na matéria seca da parte aérea da garapa variou

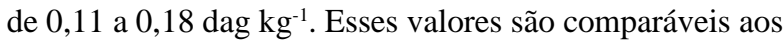
obtidos em Acacia holocericea $\left(0,12\right.$ a $\left.0,17 \mathrm{dag} \mathrm{kg}^{-1}\right)$ (BALIEIRO et al., 2001) e angico-branco $\left(0,12\right.$ a $\left.0,14 \mathrm{dag} \mathrm{kg}^{-1}\right)$ (GOMES et al., 2004), em diferentes níveis de calagem.

\section{CONCLUSÕES}

1. A resposta de mudas de garapa a fósforo $(\mathrm{P})$ varia em função do nível de calagem realizado, sendo o nível crítico de $\mathrm{P}$ no solo e na planta menor quanto maior for a saturação por bases.

2. Diante dos resultados, recomenda-se, para a formação de mudas de garapa, a aplicação de $54 \mathrm{mg} \mathrm{dm}^{-3}$ de $P$, juntamente com a aplicação de calcário que eleve a saturação por bases a $60 \%$, uma vez que nesse nível de calagem foi obtida a maior produção de MSPA.

\section{REFERÊNCIAS}

ALVAREZ V., V.H. Equilíbrio de formas disponíveis de fósforo e enxofre em dois Latossolos de Minas Gerais. 1974. 125p. Dissertação (Mestrado em Solos e Nutrição de Plantas) - Universidade Federal de Viçosa, Viçosa - MG.
ARAÚJO, A.P. Eficiência vegetal de absorção e utilização de fósforo, com especial referência ao feijoeiro. In: NOVAIS, R.F.; ALVAREZ V., V.H.e SCHAEFER, C.E.G.R. (Eds.). Tópicos em ciência do solo. Viçosa, Sociedade Brasileira de Ciência do Solo, 2000. v.1, p.163-212.

BALIEIRO, F.C.; OLIVEIRA, I.G.; DIAS, L.E. Formação de mudas de Acacia holocericea: resposta à calagem, fósforo, potássio e enxofre. Revista Árvore, Viçosa, v.25, n.2, p.183-192, 2001.

BARROS, N.F.; NOVAIS, R.F.; NEVES, J.C.L.; GOMES, J.M. Interpretação de análises químicas de solo para o crescimento de Eucalyptus spp. Revista Árvore, Viçosa, v.6, n.1, p.38-44, 1982.

BERNARDINO, D.C.S.; PAIVA, H.N.; NEVES, J.C.L.; GOMES, J.M.; MARQUES, V.B. Influência da saturação por bases e da relação $\mathrm{Ca}: \mathrm{Mg}$ do substrato sobre o crescimento inicial de jacarandá-da-Bahia (Dalbergia nigra (Vell.) Fr. All. ex Benth.). Revista Árvore, viçosa, v.31, n. 4, p. $567-473,2007$. 
BERNARDINO, D.C.S.; PAIVA, H.N.; NEVES, J.C.L.; GOMES, J.M.; MARQUES, V.B.

Crescimento e qualidade de mudas de Anadenanthera macrocarpa (Benth.) em resposta à saturação por bases do substrato. Revista Árvore, Viçosa, v.29, n.6, p.863-870, 2005.

BRAGA, J.M.; DEFELIPO, B.V. Determinação espectrofotométrica de fósforo em extratos de solos e plantas. Revista Ceres, Viçosa, v.21, n.113, p.73-85, 1974.

BRANDÃO, L.G. Desafio florestal brasileiro. Silvicultura, São Paulo, v. 18, n.73, p.23-29, 1997.

CARVALHO, P.E.R. Espécies florestais brasileiras: recomendações silviculturais, potencialidades e uso da madeira. Colombo: EMBRAPA - CNPF; Brasília: EMBRAPA - SPI, 1994. 640p.

CECONI, D.E.; POLETTO, I.; LOVATO, T.; MUNIZ, M.F.B. Exigência nutricional de mudas de erva-mate (Ilex paraguariensis A. St.-Hil.) à adubação fosfatada. Revista Ciência

Florestal, Santa Maria, v.17, n.1, p.25-32, 2007.

CECONI,D.E.; POLETTO, I.; BRUN, E.J.; LOVATO, T. Crescimento de mudas de açoita-cavalo (Luehea divaricata Mart.) sob influência da adubação fosfatada. Revista Cerne, Lavras, v.12, n.3, p.292-299, 2006.

CLARKSON, D.T. Adaptações morfológicas e fisiológicas das plantas a ambientes de baixa fertilidade. In: ROSAND, P. C. (Ed.).

Reciclagem de nutrientes e agricultura de baixos insumos nos trópicos. Ilhéus: CEPLAC/SBCS, 1985. p.45-75.

COSTA, J.P.V. Fluxo difusivo de fósforo e de potássio em Latossolos. 1998. 67p. Tese (Doutorado em Solos e Nutrição de Plantas) Universidade Federal de Viçosa, Viçosa - MG.

CRUZ, C.A.F.; PAIVA, H.N.; GOMES, K.C.O.; GUERRERO, C.R.A. Efeito de diferentes níveis de saturação por bases no desenvolvimento e qualidade de mudas de ipê-roxo ( Tabebuia impetiginosa (Mart.) Standley). Revista Scientia Forestalis, Piracicaba, n.66, p.100-107, dez. 2004.
DIAS, L.E.; ALVAREZ V., V.H.; JUCKSCH, I.; BARROS, N.F.; BRIENZA JR., S. Formação de mudas de taxi-branco (Sclerolobium paniculatum Voguel). I. Resposta a calcário e fósforo.

Pesquisa Agropecuária Brasileira , Brasília, v.26, n.1, p.69-76, 1991.

DUBOC, E.; VENTURIN, N.; VALE, F.R.; DAVIDE, A.C. Fertilização de plântulas de Copaifera langsdorffii Desf. (óleo copaíba). Revista Cerne, Lavras, v.2, n.2, p.31-47, 1996.

EMPRESA BRASILEIRA DE PESQUISA AGROPECUÁRIA (EMBRAPA). Centro Nacional de Pesquisa de Solos. 2 ed. Manual de métodos de análise de solo. Rio de Janeiro: CNPS, 1997. 212p.

EUCLYDES, R.F. Sistema para análises estatísticas e genéticas (SAEG) - manual provisório. Viçosa, CPD/UFV, divisão de pesquisa e desenvolvimento, 1983, 74p.

FERNANDES, A.R.;PAIVA,H.N.; CARVALHO, J.G; MIRANDA, J.R.P. Crescimento e absorção de nutrientes por mudas de freijó (Cordia goeldiana Huber) em função de doses de fósforo e de zinco. Revista Árvore, Viçosa, v.31,n.4, p.599-608, 2007.

FERNÁNDEZ, J.Q.P.; RUIVO, M.L.P.; DIAS, L.E.; COSTA, J.P.V.; DIAS, R.R. Crescimento de mudas de Mimosa tenuiflora submetidas a diferentes níveis de calagem e doses de fósforo, potássio e enxofre.

Revista Árvore, Viçosa, v.20, n.4, p.425-431, 1996.

FURTINI NETO, A.E. Eficiência nutricional, cinética de absorção e frações

fosfatadas em Eucalyptus spp. 1994. 99p.

Tese (Doutorado em Solos e Nutrição de Plantas) - Universidade Federal de Viçosa, Viçosa - MG.

GARCIA, N.C.P. Efeitos da calagem e de níveis de fósforo sobre o crescimento e composição mineral de mudas de cedro (Cedrela fissilis Vell.). 1986. 40p. Dissertação (Mestrado em Solos e Nutrição de Plantas) Universidade Federal de Viçosa, Viçosa - MG.

GOMES, K.C.O.; PAIVA, H.N.; NEVES, J.C.L.; BARROS, N.F.; SILVA, S.R. Influência da saturação por bases e do fósforo no crescimento de mudas de angico-branco. Revista Árvore, Viçosa, v.28, n.6, p.785-792, 2004. 
GONÇALVES, J.L.M et al. Produção de mudas de espécies nativas: substrato, nutrição, sombreamento e fertilização. In: GONÇALVES, J. L. M.;

BENEDETTI, V. (Eds.). Nutrição e fertilização florestal. Piracicaba: IPEF, 2000. p.309-350.

LORENZI, H. Árvores brasileiras. Nova Odessa: Editora Plantarum, 1992. 352p.

MARSCHNER, H. Mineral nutrition of higher plants. 2.ed. New York: Academic Press, 1995. 889p.

MARSCHNER, H.; KIRKBY, E.A.; CAKMAK, I. Effect of mineral nutritional status on shoot-root partioniting of photoassimilates and cycling of mineral nutrients. Journal of Experimental Botany, Oxford, v.47, p.1255-1263, 1996. Special Issue SI.

MEIRA NETO, J.A.A.; SILVA, A.F. Caracterização dos fragmentos florestais das áreas de influência e diretamente afetada da UHE de Pilar, Vale do rio Piranga, Zona da Mata de Minas Gerais. Viçosa: FUNARBE Universidade Federal de Viçosa, 1995. 57p.

MEIRA NETO, J.A.A.; SOUZA, A.L.; SILVA, A.F.; PAULA, A. Estrutura de uma floresta estacional semidecidual submontana em área diretamente afetada pela usina hidrelétrica de Pilar, Ponte Nova, Zona da Mata de Minas Gerais. Revista Árvore, Viçosa, v.21, n.3, p.337-344, 1997.
MEIRA NETO, J.A.A. Estudos florísticos, estruturais e ambientais nos estratos arbóreo e herbáceo-arbustivo de uma floresta estacional semidecidual em Viçosa, MG. 1997. 155p. Tese (Doutorado em Biologia Vegetal) - Universidade Estadual de Campinas, Campinas - SP.

PASSOS, M.A.A. Efeito da calagem e de fósforo no crescimento inicial da algaroba (Prosopis juliflora (SW) DC). 1994. 57p. Tese (Doutorado em Fitotecnia) -

Universidade Federal de Viçosa, Viçosa - MG.

SILVA, S.R. Crescimento de eucalipto influenciado pela compactação de solos e doses de fósforo e de potássio. 2000. 97p. Dissertação (Mestrado em Fitotecnia) Universidade Federal de Viçosa, Viçosa - MG.

SILVA, C.A.; RANGEL, O.J.P.; BELIZÁRIO, M.H. Interação calagem-adubação fosfatada e sua influência nos níveis críticos de $\mathrm{P}$ e crescimento de eucalipto. Revista Scientia Forestalis, Piracicaba, n.73, p.63-72, mar. 2007.

REIS, M.G.F.; REIS, G.G.; LELES, P.S.S.; NEVES, J.C.L.; GARCIA, N.C.P. Exigências nutricionais de mudas de Dalbergia nigra (Vell.) Fr. Allem (Jacarandá-da-Bahia) produzidas em dois níveis de sombreamento. Revista Árvore, Viçosa, v.21, n.4, p.463-471, 1997. 